\title{
Eight and a half syndrome from multiple sclerosis
}

\begin{abstract}
A 50 year old woman with a past medical history of hypertension presented with sudden onset of one-and-a-half syndrome on top of a sub-acute 7th nerve palsy. Her MRI findings showed several contrast enhancing lesions including a pontine lesion. The lumbar puncture (LP) showed oligoclonal bands. Multiple sclerosis (MS) was diagnosed, with the rare initial presentation of eight-and-a-half syndrome. The patient was treated with intravenous steroids, then placed on a disease modifying agent. In this article, we discuss the patient's imaging and review the pathology of eight-and-a-half syndrome.
\end{abstract}

Keywords: Ophthalmoplegia, OligoclonalBands, Autoimmune, Eight-and-a-Half Syndrome, Multiple Sclerosis

\author{
Volume 3 Issue 4 - 2015
}

William Jens, Aiesha Ahmed

Department of Neurology, Penn State College of Medicine, USA

Correspondence: Aiesha Ahmed, MD, Penn State College of Medicine, Hershey Medical Center, Department of NeurologyEC37, 3 Hope Drive, Hershey, PA 1733, USA, Tel 7|7-53I-1377, Fax 717-53I-0384, Email aahmedI@hmc.psu.edu

Received: October 30, 2015 | Published: December 07, 2015

\section{Introduction}

This case illustrates a lesion in a peculiar location resulting in a diagnosis of eight-and-a-half syndrome, a clinical syndrome affecting eye movements and facial nerve. Despite the known association between MS and cranial nerve lesions, the presentation of eightand-a-half syndrome is quite rare, and many clinicians have not seen such a case. Our report aims to raise awareness of eight-anda-half syndrome, as early recognition can accelerate diagnosis and management of the underlying MS.

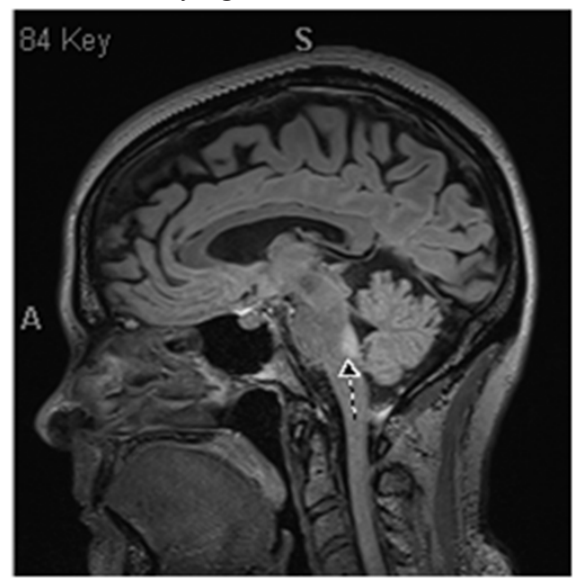

Figure I Sagittal cross section illustrating TI with contrast hyperintensity of the ponsindicating in the area of the right MLF, abducensand facial colliculus.

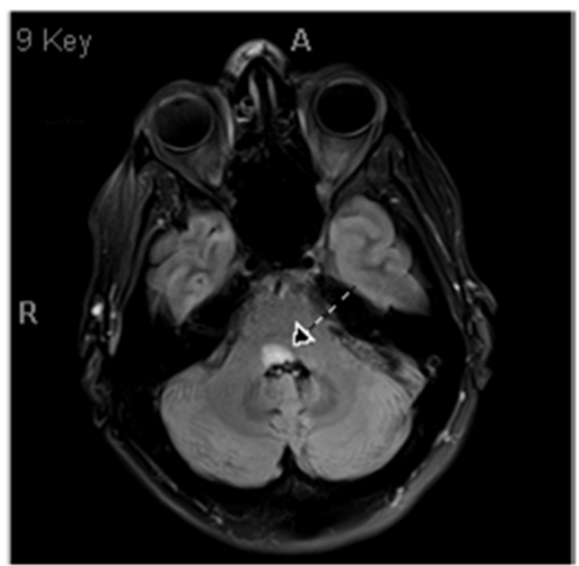

Figure 2 Axial cross section illustrating TI with contrast hyperintensity of the ponsindicating in the area of the right MLF, abducensand facial colliculus.

\section{Case description}

A 50 year old female was sent to the emergency department directly from an ophthalmology clinic with continued vertigo, facial droop with numbness and ophthalmoplegia. She had recently been hospitalized for peripheral vertigo attributed to dehydration and gastroenteritis. At that time, MRI imaging showed a small lesion in the right cerebellum of unknown source, presumed insignificant. She improved with fluids and was discharged to her home. A few days after discharge,she noticed a slight right facial droop and altered taste on the right side of her tongue. Her primary care doctor diagnosed her with Bell's palsy and placed her on a course of steroids followed by doxycycline. Her symptoms became worse despite treatment. She developed blurred vision, which led to the ophthalmology clinic appointment.

The emergency department consulted neurology. The physical exam showed right gaze limitation and left eye limitation of adduction with horizontal nystagmus on abduction ("one-and-a-half syndrome"). She had right-sided lower motor neuron facial nerve palsy with a mild taste difference on the right anterior tongue. She was also found to be ataxic and had a positive Rhomberg sign. MRI (Figures 1\&2) showed an enhancing area in the floor of the 4th ventricle at the pons without restricted diffusion as well as two small, stable, areas of FLAIR hyperintensity seen in the right cerebral white matter and cerebellum. Under the McDonald criteria she had probable MS. Cerebrospinal fluid analysis was performed to confirm the diagnosis and found to show two nucleated cells, normal protein,normal glucose, and $>5$ oligoclonal bands. Based on the clinical symptoms and other diagnostic testing findings, steroid therapy was started for MS. One month later the patient was started on glatiramer acetate for preventive therapy.

\section{Discussion}

This case illustrates a rare presentation of MS causing eight-anda-half syndrome. MS symptoms typically originate from the optic nerve, spinal cord or cortical lesions. ${ }^{1} \mathrm{MS}$ is an autoimmune disease that damages the myelin coating and to the nerve fibers themselves, which interferes with the transmission of nerve signals between the brain, spinal cord and the rest of the body. ${ }^{2}$ Disrupted nerve signals cause the symptoms of MS, which vary from one person to another and over time for any given individual, depending on where the damage occurs. ${ }^{3}$ There have been previous reports of MS causing an eight-and-a-half. In this case, the imaging highlights the anatomy behind this rare syndrome. ${ }^{1,4,5}$ 
Lesions affecting eye movements can be difficult to localize, but understanding the relevant anatomy and interconnections simplifies the process. Six extraocular muscles control the motions of each eye: the medial and lateral rectus produce lateral motion, the superior and inferior rectus produce vertical movement, and the superior oblique and the inferior oblique muscles make torsional globe movements. ${ }^{6}$ The oculomotor nerve supplies the levator palpebrae superioris muscles, superior, medial and rectus, as well as the inferior oblique muscles, the trochlear nerves innervates the superior oblique muscles while the lateral rectus muscles is innervated by the abducens. ${ }^{7}$ Horizontal conjugate gaze involves the coordination of the Paramedian Pontine Reticular Formation (PPRF), Medial Longitudinal Fasciculus (MLF), abducens and oculomotor nuclei. ${ }^{8}$ The PPRF is located near the near the abducens nucleus at the level of the facial colliculus. The PPRF receives afferent information from the frontal and parietal eye fields and innervates ipsilateral abducens nucleus. The abducens nucleus innervates the ipsilateral lateral rectus and simultaneously innervates the contralateral oculomotor nucleus via the MLF resulting in medial rectus activation. ${ }^{9}$ One-and-a-half syndrome is a clinical disorder characterized by conjugate horizontal gaze palsy in one direction and an inter nuclear ophthalmoplegia in the other. ${ }^{10}$ It results from a single unilateral lesion of the paramedian pontine reticular formation or the abducens nucleus, with interruption of internuclear fibers of the ipsilateral medial longitudinal fasciculus after crossing the midline. ${ }^{11}$ Anatomically the facial colliculus is just below this area and if the lesion extends to this region, it results in combining one-and-a-half syndrome and facial nerve palsy, a very rare syndrome, called eightand-a-half syndrome. ${ }^{12}$ Known causes include MS, stroke, and cancer. Treatment and prognosis of eight-and-a-half syndrome depend upon the underlying cause, but typical recovery occurs within the first few months. ${ }^{13}$

In a review of the literature via OVID, a search for "eight-anda-half syndrome" resulted in 69 results, of which 26 articles were deemed relevant by the author. Among the cases reported in these articles, ischemic stroke was the most commonly identified cause of eight-and-a-half syndrome (18 cases). Other cases of eight-and-ahalf syndrome were associated with autoimmune causes (four cases), hemorrhagic stroke (three cases) giant cell arteritis (one case), and pontine tuberculoma (one case). In the literature, eight-and-a-half syndrome was also described as occurring in conjunction with related syndromes. Reports of "nine syndrome" described patients with eight-and-a-half syndrome and an additional lesion in the midbrain tegmentum/red nucleus causing contralesional hemiataxia. ${ }^{14}$ Other related syndromes included " $15 \frac{1}{2}$ syndrome" (patient with bilateral seventh nerve palsy and one-and-a-half syndrome) and " 16 syndrome" (patient with bilateral seventh nerve palsy and hemiparesis and oneand-a-half syndrome) ${ }^{15}$ In an interesting case, a patient's Holmes and palatal tremor and eight-and-a-half syndrome were the results of an injury in the Guillain-Mollaret triangle (composed of the contralateral dentate nucleus, the ipsilateral red and inferior olivary nucleus). ${ }^{16}$

\section{Conflicts of interest}

None.

\section{References}

1. Skaat A, Huna-Baron R. Eight-and-a-half syndrome: a rare pontine neuro-ophthalmologic syndrome. Arch Neurol. 2012;69(7):934-935.

2. Messina S, Patti F. Gray matter in multiple sclerosis: cognitive impairment and structural MRI. Mult Scler Int. 2014;2014:609694.

3. Karussis D. The diagnosis of multiple sclerosis and the various related demyelinating syndromes: a critical review. J Autoimmun. 2014;4849:134-142

4. Mortzos P, Nordling MM, Sorenson TL. Eight-and-a-half syndrome as presenting sign of childhood multiple sclerosis. $J$ AAPOS. 2014;18(5):490-492

5. Bocos-Portillo J, Ruiz Ojeda J, Gomez-Beldarrain M, et al. Eightand-a-Half Syndrome. JAMA Neurol. 2015;72(7):830.

6. Blumenfeld H. Brainstem II: eye movements and pupillary control. In: Blumenfeld H (Ed.), Neuroanatomy through clinical cases. (1st edn), Sinauer Associates, Sunderland, Mass, USA. pp.2002.529-574.

7. Wichmann W, Müller-Forell W. Anatomy of the visual system. Eur J Radiol. 2004;49(1):8-30.

8. Bae YJ, Kim JH, Choi BS, et al. Brainstem pathways for horizontal eye movement: pathologic correlation with MR imaging. Radiographics. 2013;33(1):47-59.

9. Karatas M. Internuclear and supranuclear disorders of eye movements: clinical features and causes. Eur J Neurol. 2009;16(12):1265-1277.

10. Martyn CN, Kean D. The one-and-a-half syndrome: clinical correlation with a pontine lesion demonstrated by nuclear magnetic resonance imaging in a case of multiple sclerosis. $\mathrm{Br} J$ Ophthalmol. 1988;72(7):515-517.

11. Eggenberger E. Eight-and-a-half syndrome: one-and-a-half syndrome plus cranial nerve VII palsy. J Neuroophthalmol. 1998;18(2):114-116.

12. Wall M, Wray SH. The one-and-a-half syndrome-a unilateral disorder of the pontine tegmentum: a study of 20 cases and review of the literature. Neurology. 1983;33(8):971-980.

13. Nandhagopal R1, Krishnamoorthy SG. Eight-and-a-half syndrome. $J$ Neurol Neurosurg Psychiatry. 2006;77(4):463.

14. Rosini F, Pretegiani E, Guideri F, et al. A Eight and a half syndrome with hemiparesis and hemihypesthesia: the nine syndrome? J Stroke Cerebrovasc Dis. 2013;22(8):637-638.

15. Connors R, Ngan V, Howard J. A case of complete lateral gaze paralysis and facial diplegia: the 16 syndrome. $J$ Neuroophthalmol. 2012;33(1):69-70

16. Bolen RD, Balakrishnan N. Palatal myoclonus, eight-and-a-half syndrome, and Holmes tremor in a patient from a single brainstem lesion. J Neurol Sci. 2014;347(1-2):411-412.

\section{Acknowledgments}

None. 